
Advanced Biomaterials-Characterization, Tissue Engineering and Assemb1y, MRS Proceedings,
Vo1. 711, S.C. Moss (editor), 2002.

\title{
Synchrotron X-ray Observations of a Monolayer Template for Mineralization
}

\author{
E. DiMasi ${ }^{1 *}$ and L. B. Gower ${ }^{2}$
}

${ }^{1}$ Dept. of Physics, Brookhaven National Laboratory, Upton NY 11973

${ }^{2}$ Dept. of Materials Science and Engineering, University of Florida, Gainesville FL 32611

${ }^{*}$ Corresponding author: dimasi

\begin{abstract}
Mineral nucleation at a Langmuir film interface has been studied by synchrotron x-ray scattering. Diluted calcium bicarbonate solutions were used as subphases for arachidic and stearic acid monolayers, compressed in a Langmuir trough. Self-assembly of the monolayer template is observed directly, and subsequent crystal growth monitored in-situ.
\end{abstract}

\section{INTRODUCTION}

A persistent question in biomineralization concerns the interactions between the organic molecules and the nucleating mineral. Since mineralization often occurs in proximity to an organic matrix having structural regularities that match the mineral lattice spacings, the organic material is assumed to act as a template for nucleation. ${ }^{1}$ However, a number of recent experiments emphasize that mineralization can also proceed through amorphous presursors, which subsequently crystallize. ${ }^{2,3}$ In these systems, the importance of registry with a macromolecular template is called into question.

One important model system that addresses this issue consists of a surfactant monolayer assembled at the air-water interface. These molecules are well known to form ordered, two-dimensional templates, typically with centered rectangular or hexagonal lattices. ${ }^{4}$ With the right choice of surfactant, the intermolecular distances, headgroup charges, and so on can be systematically varied. The advantages of these tunable systems have been demonstrated in the study of calcium carbonate nucleation, where calcite or vaterite formation with specific crystal size distribution, habit, and orientation at the surface was observed in the presence of various surfactants. ${ }^{5}$ Similar work has been pursued with other minerals. ${ }^{6,7}$

In the works cited above, crystal morphology was assessed by optical and electron microscopy. These observations were not sensitive to the microscopic arrangement of surfactant molecules and cations, nor could they directly measure crystal growth during its earliest stages. In fact, this has been a common limitation for other studies of biomineralizing systems: detailed measurements of lattice spacings of mineral and template during early growth times have not been available. Similarly, the potential for mineralization to proceed through metastable phases such as amorphous or hydrated precursors can not always be assessed.

In this paper we describe synchrotron x-ray scattering studies of calcium carbonate nucleating from solution at fatty acid monolayers. Our aim is to give an overview of the structural information that can be directly obtained by this method. Possible routes to mineralization in this system are illustrated in Figure 1. First, it is expected that the 


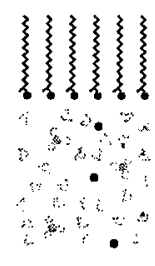

a

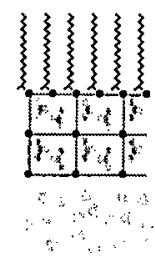

b

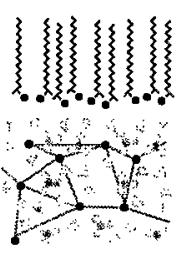

C

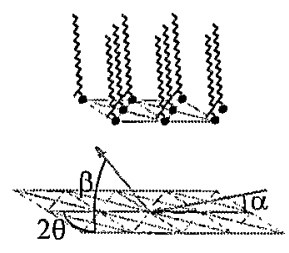

d

Figure 1: (a) Fatty acid monolayer assembled on an aqueous calcium bicarbonate subphase. The negatively charged headgroups attract a layer of bound cations. The monolayer may serve as a template for direct nucleation of crystallites (b), or as a boundary surface that collects species for nucleation through another route, such as an amorphous precursor phase (c). (d) View of ordered two-dimensional lattice of surfactant molecules, and geometry for $\mathrm{x}$-ray scattering measurements.

charged headgroups will attract a layer of cations (Figure 1(a)). If the lattice spacings of the surfactant and mineral structures match closely, crystals may form directly at the template (Figure 1(b)). It is also possible that the monolayer does not interact strongly with the nucleating minerals. It may instead simply form a physical boundary that collects the mineral constituents, which may then nucleate through various routes including metastable hydrated and/or amorphous phases. In the following sections we will describe how x-ray scattering methods can probe the structure of the nucleating system.

\section{X-RAY SCATTERING METHODS}

Two surface scattering techniques, x-ray reflectivity and grazing incidence diffraction, provide complementary structural information. With x-rays incident onto the sample at an angle $\alpha$ (Figure $1(\mathrm{~d})$ ), intensity can be detected at reflection angle $\beta$ and in-plane scattering angle $2 \theta$. The momentum transfer components within and normal to the layer plane, which relate the scattering angles to scattered intensity in reciprocal space, are given by

$$
q_{x y}=(2 \pi / \lambda)\left[\cos ^{2} \alpha+\cos ^{2} \beta-2 \cos \alpha \cos \beta \cos (2 \theta)\right]^{1 / 2} ; q_{z}=(2 \pi / \lambda)[\sin \alpha+\sin \beta]
$$

respectively. ${ }^{8}$

Reflected beams from the air-monolayer and monolayer-water interfaces give rise to an interference pattern which can be analyzed to determine the nature of those interfaces. The reflectivity is defined as the ratio of detected to incident intensity, for $\alpha=\beta$ and $2 \theta=0$ and is related to the electron densities of the scattering layers: in the Born approximation, the reflectivity depends on the Fourier transform of the derivative of the density profile $\rho(z)$,

$$
R\left(q_{z}\right)=R_{F}\left(q_{z}\right)\left|\left(1 / \rho_{w}\right) \int_{-\infty}^{+\infty} d z(d \rho / d z) \exp \left(i q_{z} z\right)\right|^{2}
$$

where $R_{F}\left(q_{z}\right)$ is the Fresnel reflectivity and $\rho_{w}$ is the electron density of water. To determine the structure from the reflectivity measurement, a model function $\rho(z)$ is parameterized, based upon physical features of the system such as the hydrocarbon tails 
and headgroups. The scattering pattern calculated from the model is refined against the data, in a manner analogous to the standard methods of crystallography, allowing us to obtain the monolayer thickness, density, and so on.

Structure within the layer plane, such as ordered rows of surfactant molecules or the atomic planes of underlying mineral crystals, will give rise to Bragg peaks which are detected as a function of the in-plane momentum transfer $q_{x y}$. This intensity can be detected if the incident angle $\alpha$ is kept within the critical angle for total external reflection of x-rays. This limits the $\mathrm{x}$-ray penetration to $<50 \AA$, illuminating primarily the near-surface region. Scattered intensity from a strictly two-dimensional structure would be detected entirely within the layer plane $(\beta \approx 0)$. Intensity will also be observed at finite $\beta$ values, in the case of ordered tilting of the hydrocarbon tails. ${ }^{4}$

\section{EXPERIMENT}

Supersaturated calcium bicarbonate solutions were prepared by dissolving calcium carbonate powder in purified water $\left(2 \mathrm{~g} \mathrm{CaCO}_{3} / 800 \mathrm{ml}\right)$ with bubbled $\mathrm{CO}_{2}$ gas. The solution was diluted to reduce the crystal nucleation rate, poured into the Langmuir trough, and chilled to $18^{\circ} \mathrm{C}$. Arachidic acid $\left(\mathrm{CH}_{3}\left(\mathrm{CH}_{2}\right)_{18} \mathrm{COOH}\right)$ and stearic acid $\left(\mathrm{CH}_{3}\left(\mathrm{CH}_{2}\right)_{16} \mathrm{COOH}\right)$ monolayers were spread from chloroform solution. After sample preparation, the trough was closed within a gas tight box, and $\mathrm{x}$-ray measurements made under helium atmosphere, with wavelength $\lambda=1.556 \AA$. X-ray experiments utilized the Harvard/BNL liquid surface spectrometer at beamline X22B at the National Synchrotron Light Source at Brookhaven National Laboratory. A Bicron single channel detector was used for the reflectivity. Grazing incidence diffraction measurements utilized a linear detector with a vertical acceptance of $10^{\circ}$ and a horizontal resolution determined by Soller slits. These methods have been described previously. ${ }^{9}$

\section{RESULTS}

The assembly of the ternplate and the collection of cations at the interface comprise the first stages of nucleation. To show how the surface pressure and the presence of the cations affect the monolayer, we compare Langmuir film structures of arachidic acid under three different conditions: low surface pressure on pure water; compressed to the highest maintainable surface pressure on pure water; and spread at low pressure on a saturated calcium bicarbonate solution diluted 1:1 with water. Figure 2(a) shows intensity contours for in-plane peaks, measured for arachidic acid on water with a low surface pressure of $P=8 \mathrm{mN} / \mathrm{m}$. Two diffraction peaks are observed, one having a nonzero $q_{z}$ component, which indicates that the hydrocarbon tails are tilted. This pattern arises from a centered rectangular unit cell with dimensions $a=5.23 \AA$ and $b=8.56 \AA$, or an area of $22.4 \AA^{2} /$ molecule. The hydrocarbon tails tilt along the $b$ axis at an angle of $26.5^{\circ}$ to the surface normal. When the film is compressed to pressures above $25 \mathrm{mN} / \mathrm{m}$, only one in-plane peak is observed, as shown in Figure 2(b). The corresponding structure is an untilted hexagonal phase with $a=b / \sqrt{3}=4.75 \AA$, or an area of $19.6 \AA^{2} /$ molecule. These observations are in agreement with literature values for arachidic acid films under these conditions. ${ }^{4,10}$ By contrast, when arachidic acid is spread onto the calcium bicarbonate 

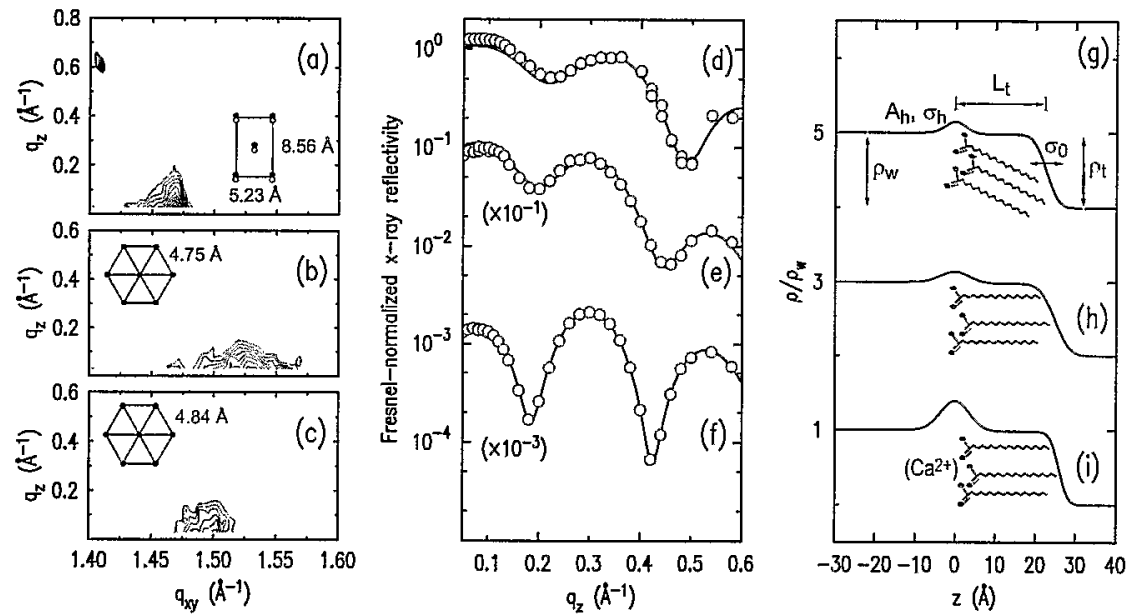

Figure 2: Left: Intensity contours of in-plane diffraction peaks from arachidic acid Langmuir films. Insets: planar unit cells determined from the diffraction data. Solid and doubled circles represent untilted and tilted molecules respectively. Center panel, open circles: X-ray reflectivity data, normalized to the Fresnel reflectivity of water. Solid lines: fits from model density profiles. Right: model density profiles (shifted for clarity) and the corresponding physical structures. (a),(d),(g) Pure water subphase, $P=8 \mathrm{mN} / \mathrm{m}$. (b),(e),(h) Pure water subphase, $P>25 \mathrm{mN} / \mathrm{m}$. (c),(f),(i) Diluted calcium bicarbonate subphase, $P=8 \mathrm{mN} / \mathrm{m}$.

subphase, untilted domains self-assemble even at low pressure. Although the nominal area calculated from the trough surface area and surfactant density is $22.7 \AA^{2}$ per molecule and the film was not compressed, the diffraction peaks in Figure 2(c) show that ordered domains have formed. The lattice constants $a=b / \sqrt{3}=4.84 \AA$ imply an area of $20.4 \AA^{2} /$ molecule, only slightly larger than that of the high-pressure film on pure water. This observation is important because previous studies have sometimes assumed that the effect of applied pressure is to make fine adjustments to the intermolecular spacings. In the presence of the cations, this is not the case.

Reflectivity data measured under these conditions are shown in Figure 2(d)-(f) (open circles). (Data have been normalized to the Fresnel reflectivity and shifted for clarity.) The oscillations are due to interference between x-rays reflecting from the molecule-air and molecule-water interfaces. For arachidic acid on pure water, the two data sets are similar except for a shift of the minima to lower $q_{z}$ for the high pressure condition (Figure 2(e)). This reflects the straightening of the molecular tilt, which makes the surfactant layer thicker along the surface normal. The reflectivity data for the calcium bicarbonate 
subphase show a further shift in $q_{z}$, as well as a dramatic increase in the oscillation amplitude (Figure 2(g)). Both of these features can be attributed to the incorporation of $\mathrm{Ca}$ ions at the headgroups, which increases the electron density significantly.

Fits to the data (solid lines) allow us to determine length scales and densities along the surface-normal direction. The model electron density profiles, shown in Fig. 2(g)-(i), are normalized to that of water, $\rho_{w}=0.33$ electrons $/ \AA^{3}$. The electron density of the packed hydrocarbon tails is fixed to the known value $\rho_{t}=0.98 \rho_{w} .{ }^{8}$ Parameters varied for the fits shown are the tail length $L_{t}$, headgroup Gaussian amplitude $A_{h}$ and width $\sigma_{h}$, and a smearing at the vapor interface characterized by a Gaussian derivative of width $\sigma_{0}$. Fits were explored in which different combinations of parameters were varied, and gave sirnilar results; smearing by thermal surface waves and the restricted $q_{z}$ range of the measurements limit the extent to which fine structural details can be distunguished. The following description, while not unique, captures the essential features of the data.

The tail length $L_{t}=22.6 \AA$ for the tilted phase, $24.8 \AA$ for the untilted phase on water, and $25.9 \AA$ on calcium bicarbonate solution. The ratio $22.6 / 24.8=0.91$ is in excellent agreement with $\cos 26.5=0.89$ corresponding to the molecular tilt obtained from in-plane diffraction. The further increase in $L_{t}$ for the calcium bicarbonate case likely arises from the incorporation of cations into the headgroup region. The interface smearing $\sigma_{0}=2-3 \AA$, typical of the thermal roughening of water and of other Langmuir films which are not too rigid. ${ }^{8}$ The Gaussian term describing the headgroup region can be described by a width $\sigma_{h} \approx 3.5 \AA$ for all three data sets. The integrated area $\left(A_{h} / \sigma_{h} \sqrt{2 \pi}\right)$ gives the excess electron density of the headgroups relative to that of the water. The data agree well with assigning the 23 electrons per $\mathrm{COOH}$ unit to a $2.7 \AA$ thick region between the tails and the pure water subphase at both pressures. In the presence of calcium carbonate, the amplitude of the Gaussian term is about twice as large. The excess electron density in the headgroup region is about 4-5 electrons per arachidic acid molecule, suggesting a density of one $\mathrm{Ca}$ ion per every four or five molecules. This cation binding seems reasonable given the near-neutral $\mathrm{pH}$, at which significant ionization of the headgroups is not expected.

Notably, our data are not consistent with the 1:1 cation/headgroup ratio implied by a mechanism of stereochemical matching. ${ }^{5}$

We now make some remarks about subsequent crystallization of the system. The data shown in Figure 2(c) and (f) were obtained within four hours of preparing the sample. At this point, macroscopic crystallites were already visible on the surface, and calcite Bragg peaks were present in the diffraction pattern. During the next 24 hours, increasing intensity at vaterite peak positions was also observed. Reflectivity measurements indicated that the monolayer was becoming rough and inhomogeneous. No evidence was found for a well-defined mineral layer beneath the template, or for coherence between monolayer and oriented crystallites.

Interestingly, these results are at odds with work by our group on compressed stearic acid monolayers (unpublished), and on experiments with poly(acrylic acid) added to the subphase (manuscript in preparation). In both of these cases, reflectivity measurements detect a coherent mineral layer that forms beneath the monolayer on 40-300 $\AA$ length scales. This layer has a low density suggestive of hydrated calcium carbonate, and subsequently crystallizes into predominantly vaterite. If such a process is occuring in the present system as well, it may be difficult to detect due to the uneven coverage of 
surfactant; rapid nucleation of calcite at the bare water surfaces certainly disrupts the homogeneity of the surrounding regions. We believe that along with the detailed structure of the monolayer template and the kinetics dictated by the solution chemistry, simple aspects such as the monolayer coverage can thus affect mineral morphology. We anticipate that synchrotron $\mathrm{x}$-ray scattering studies will be significant in obtaining further information about these systems.

\section{CONCLUSIONS}

We have shown how in-situ $x$-ray scattering studies enable direct measurements of the structure of a monolayer template during mineralization from solution. Although compression of the Langmuir film has been correlated to mineral morphology in past studies, we demonstrate that in the presence of the mineralizing species, surfactant molecules assemble into close packed domains even at low surface pressure. Diffraction is the most accurate way to obtain the molecular spacing. For calcium carbonate nucleating at an arachidic acid monolayer, $x$-ray reflectivity measurements found that the calcium binds to the monolayer at a density of one cation per four-five surfactant molecules. The low cation binding does not support the model of template directed nucleation.

\section{ACKNOWLEDGMENTS}

We acknowledge support from the Engineering Research Center (ERC) for Particle Science and Technology at the University of Florida, NSF Grant \#EEC-94-02989, and the Industrial Partners of the ERC. The National Synchrotron Light Source is supported under U.S. DOE Contract DE-AC02-98CH10886.

\section{REFERENCES}

1. P. J. J. A. Buijnsters et al, Langmuir 17, 3623 (2001).

2. G. Xu, N. Yao, I. A. Aksay, and J. T. Groves, J. Am. Chem. Soc. 120, 11977 (1998).

3. L. B. Gower and D. J. Odom, J. Crystal Growth 210, 719 (2000).

4. V. M. Kaganer, H. Möhwald, and P. Dutta, Rev. Mod. Phys. 71, 779 (1999).

5. S. Mann, B. R. Heywood, S. Rajam, and J. D. Birchall, Nature 334, 692 (1988).

6. B. R. Heywood and S. Mann, J. Am. Chem. Soc. 114, 4681 (1992).

7. B. R. Heywood and S. Mann, Langmuir 8, 1492 (1992).

8. D. K. Schwartz, M. L. Schlossman, and P. S. Pershan, J. Chem. Phys. 96, 2356 (1992).

9. H. Tostmann et al, Phys. Rev. B 59, 783 (1999).

10. K. Kjaer et al, J. Phys. Chem. 93, 3200 (1989). 